\title{
A Clinical Study to assess the Efficacy of Commonly Available Denture Adhesive Materials in Mandibular Dentures
}

\author{
Puja Malhotra, Bhupender Yadav, Harish Yadav, Sumit Singh Phukela
}

\section{ABSTRACT}

Retention in mandibular dentures has always been a challenge for the treating dentist. Denture adhesives are known to improve the adhesive bond between the denture and the underlying tissues. However, denture adhesives still remain unexplored and not much is known about the efficacy of these materials in mandibular dentures especially in patients with poor foundations. The present study was carried out to compare the efficacy of different commercially available denture adhesives in relation to their retentive ability in patients with good well formed mandibular ridges and in patients with resorbed and flat mandibular ridges. Seventy edentulous patients, age range between 50 to 70 years, were selected. The patients were divided into 2 groups; group 1 comprised of 35 patients with good well formed mandibular ridges and group 2 consisted of 35 patients having low well rounded mandibular ridges. The adhesion and cohesion that developed between the dentures and the underlying tissues when the various materials were interposed between them was evaluated with the help of a test apparatus in newtons. Mean value of retention in denture without adhesive was $9.93 \mathrm{~N}$ and $24.53 \mathrm{~N}$ in resorbed and well formed ridges respectively. Use of denture adhesive materials led to a higher value of retention as opposed to when the dentures were used without adhesives. This was true for patients with good well formed mandibular ridges as well as for patients with poor foundations. Among the various denture adhesive powders tested, Fittydent showed better results, i.e. 29.56 and 33.05 in resorbed and well-formed ridges respectively as opposed to fixon which showed values of 16.47 and 27.70 respectively.

Keywords: Adhesion, Retention, Cohesion.

How to cite this article: Malhotra P, Yadav B, Yadav H, Phukela SS. A Clinical Study to assess the Efficacy of Commonly Available Denture Adhesive Materials in Mandibular Dentures. Int J Prosthodont Restor Dent 2013;3(4):125-130.

\section{Source of support: Nil}

Conflict of interest: None

\section{INTRODUCTION}

The number of completely edentulous patients has grown many folds in the recent years probably because of the advancements in the medical fields leading to an increased geriatric population. Therefore, complete denture still forms an integral part of prosthodontic treatment options in spite of the advent and popularity of dental implants. Especially, in a country like India, where dental treatment is still primarily governed by its economics.
Retention in complete dentures is and has always been the most important issue as far as complete dentures are concerned. The situation worsens with advancing age and resorption of residual tissues. Besides, this problem of obtaining adequate retention becomes more challenging in case of mandibular dentures especially in patients with poor foundations. History has revealed, various techniques that have been devised and employed to provide satisfactory dentures to these patients. Functional impression techniques, recording of neutral zone, admix impression technique, overdentures, implants have been used in an attempt to provide satisfactory dentures to the patients. However, it has been observed that patients still resort to the use of denture adhesives or glues to gain that extra retention or comfort or confidence that an adhesive provides.

Denture adhesives occupy a very important place in prosthodontics. Although, they are commonly used and easily available dentists hesitate to recommend denture adhesives in routine dental practice probably because of the various myths associated with it. The current dental literature supports the use of denture adhesives and dispels some of the negative opinions that originate with them. A number of denture adhesives are presently in use, however their efficacy is questionable.

Hence, the aim of present study was to assess the in vivo efficacy of denture adhesives. And also to assess their role in patients with extensive resorption in mandibles.

\section{AIMS AND OBJECTIVES}

The aim of the present study was:

1. To compare the efficacy of different commercially available denture adhesives in relation to their retentive ability.

2. To assess the efficacy of denture adhesives in patients with good well-formed mandibular ridges.

3. To assess the efficacy of denture adhesives in patients with low well rounded mandibular ridges.

\section{MATERIALS AND METHODS}

Seventy edentulous patients, age range between 50 and 70 years, were selected from the outpatient Department of 
Prosthodontics, Sri Govind Tricentenary Dental College, Gurgaon. The patients were then divided into two groups: group 1 comprised of patients with good well-formed mandibular ridges or order 3 of Atwood's classification ${ }^{1}$ (Fig. 1) and group 2 consisted of patients having low well rounded mandibular ridges or order 5 of Atwood's classification $^{1}$ (Fig. 2). All the patients were apparently in good general health and did not report of any systemic disease. Finally, the selected patients were informed about the study and an informed consent was taken from them.

New set of complete denture was fabricated for all the patients following the conventional technique. On the day of the denture insertion, border extensions were checked and corrected. Occlusion was verified. All patients were then asked to use the dentures for 4 weeks prior to the start of the study, allowing them to get used to the dentures. At the end of 4 weeks, the patients were recalled and the dentures reevaluated for any soreness, etc.

The mandibular dentures were then prepared for the study; a hook of about 2 inch length was secured on the lingual surface of the denture, i.e. Just behind and between

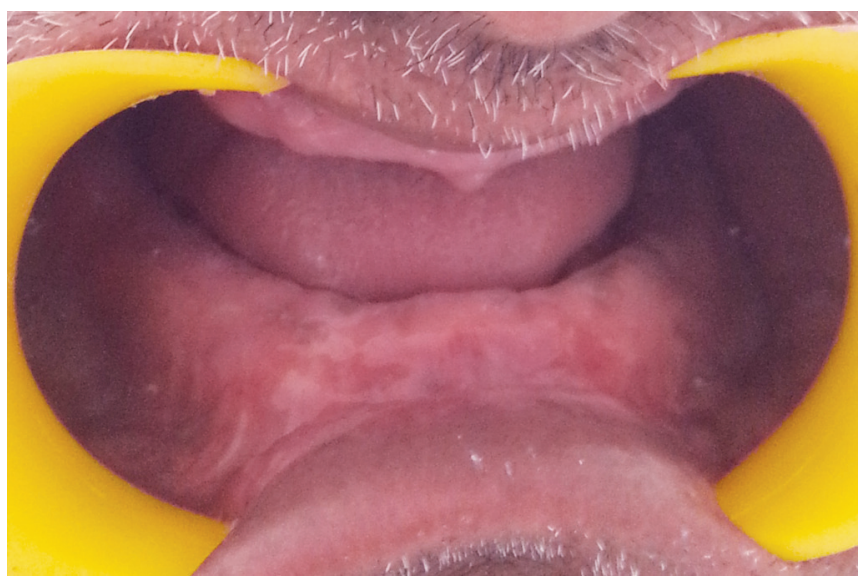

Fig. 1: Test apparatus

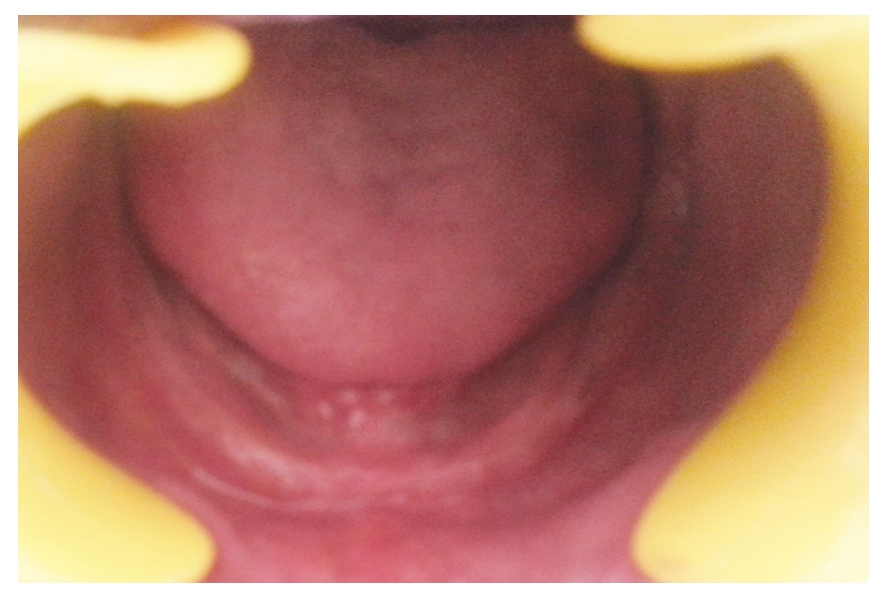

Fig. 2: Mandibular denture with attached hook mandibular central incisors with auto polymerizing resin (Fig. 3). This was done in order to apply force in the midline via the test apparatus (Fig. 4). The load will be recorded directly on the load indicator unit.

At the day of testing, patients were seated comfortably in upright position and were made to rinse his/her mouth with water. Dentures were inserted and patient were made to close in centric occlusion for 5 seconds and left in position for 2 minutes. Then after calibration of the device, the patient opened his mouth to a sufficiently comfortable distance and hook of the device engaged in the hook attached to the mandibular complete denture. The retention device was activated and force was applied steadily until the denture got completely detached from the underlying ridge, the force value at which the denture was dislodged was recorded as the retention force of the denture.

The sample group in this study was divided into two groups of 35 patients each.

- Group 1: Patients with good well-formed mandibular ridges $(\mathrm{n}=35)$.

- Group 2: Patients with low well-rounded mandibular ridges $(\mathrm{n}=35)$.

In both the groups, the experiment was carried out in three parts, i.e. the control group A comprised of retention values when no adhesive was used. Group B when fixon was interposed between the mandibular denture and underlying tissues and group $\mathrm{C}$ when fittydent was used. Therefore to summarize:

- Group 1A: Patients with good mandibular ridges, when no adhesive is used.

- Group 1B: Patients with good mandibular ridges, when fixon is used.

- Group 1C: Patients with good mandibular ridges, when fittydent is used.

- Similarly,

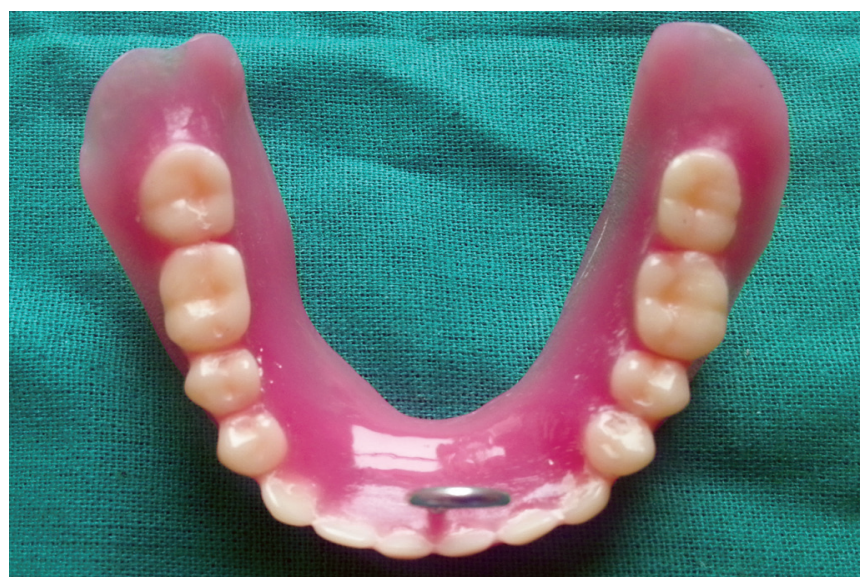

Fig. 3: Test material applied on tissue surface of denture 


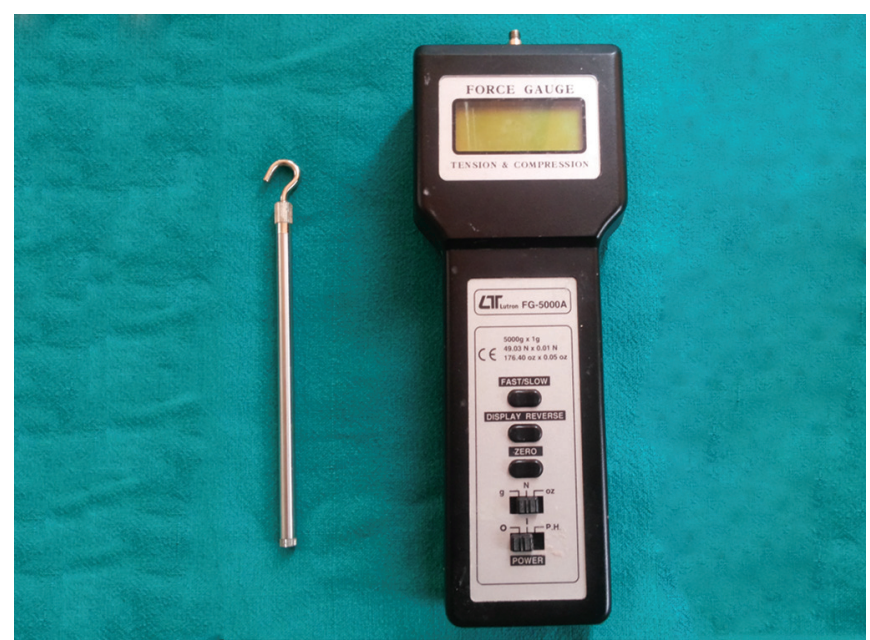

Fig. 4: High well-rounded mandibular ridge, (a) retention value in high well-rounded mandibular ridge without any test material, (b) retention value in high well-rounded mandibular ridge with fixon test material, (c) retention value in high well-rounded mandibular ridge with fittydent test material

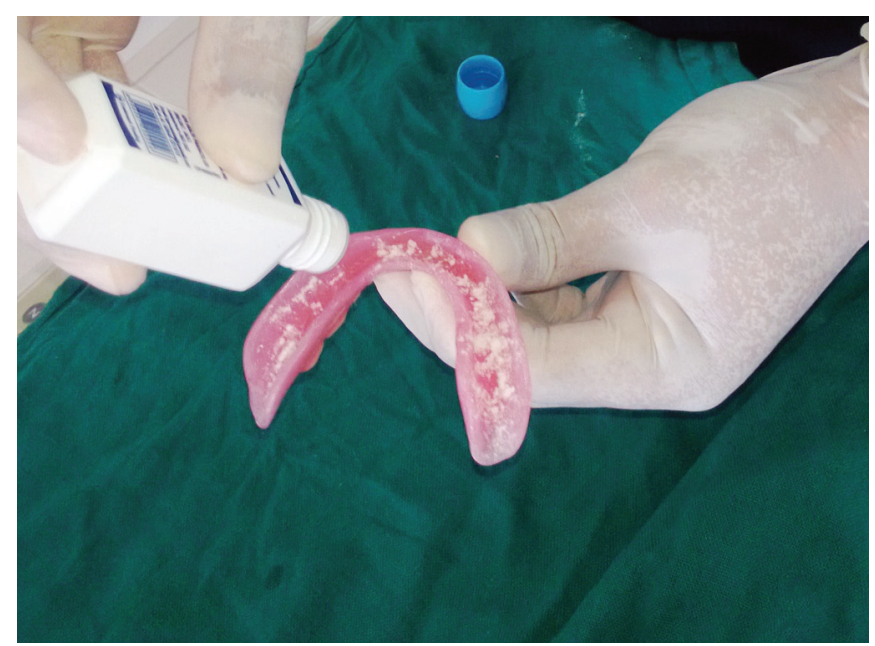

Fig. 5: Low well-rounded/resorbed mandibular ridge, (a) retention value in low well-rounded/resorbed mandibular ridge without any test material, (b) retention value in low well-rounded/resorbed mandibular ridge with fixon test material, (c) retention value in low well-rounded/resorbed mandibular ridge with fittydent test material

- Group 2A: Patients with poor mandibular ridges, when no adhesive is used.

- Group 2B: Patients with poor mandibular ridges, when fixon is used.

- Group 2C: Patients with poor mandibular ridges, when fittydent is used.

A controlled experimental procedure was followed. Each procedure was repeated three times with half an hour gap in between. The adhesive powder was sprinkled on to the wetted intaglio surface of the dentures and the excess was shaken off prior to inserting the dentures in the mouth (Fig. 5). The samples were cleaned very carefully after each experiment with an aqueous solution of detergent. They were thoroughly washed with distilled water to remove all traces of the adhesive material and then dried with clean absorbent

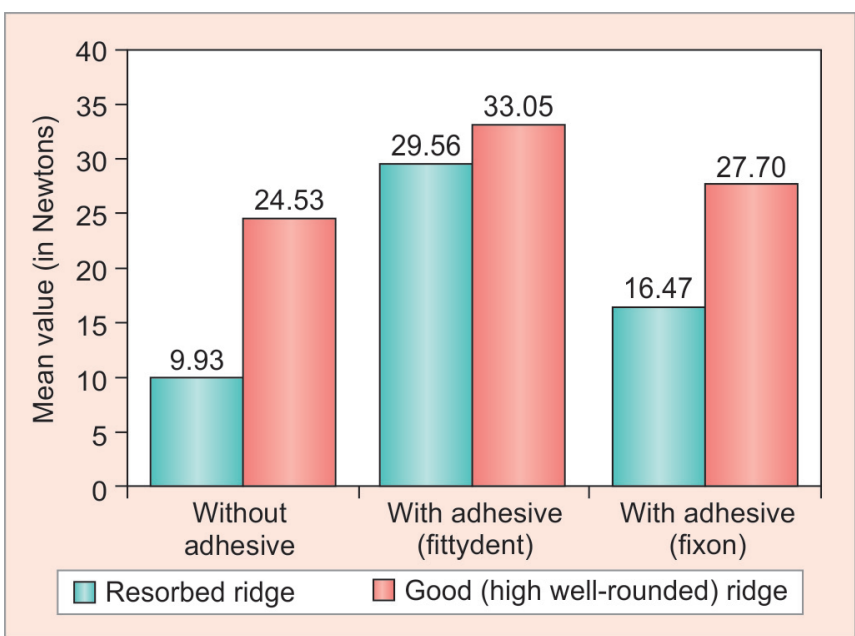

Graph 1: Comparison of two groups, i.e. resorbed ridge (low wellrounded and good (high well-rounded) ridge with respect to retention without adhesive, retention with adhesive (fittydent) and retention with adhesive (fixon)

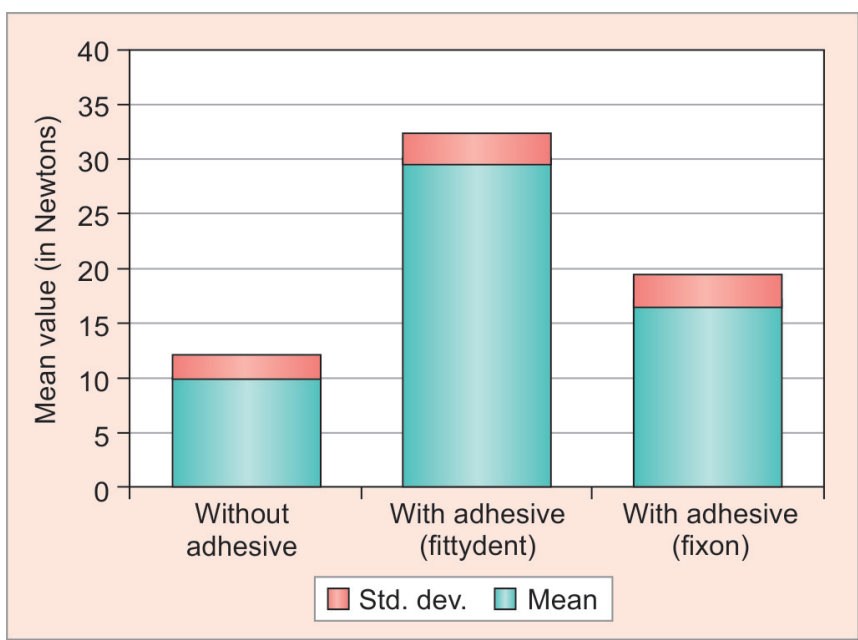

Graph 2: Comparison of retention without adhesive, retention with adhesive (fittydent) and retention with adhesive (fixon) in resorbed ridge (low well-rounded) group

tissue, so that the procedure would be free of any effects between the treatments.

\section{RESULTS}

The values of the various interfacial mediums used in the various categories are given in Table 1.

\section{DISCUSSION}

'Denture adhesive' 2 is a commercially available, non toxic, soluble material that when applied to the tissue surface of the denture enhance their retention, stability and performance. These are available as powder, paste, tape or cushion. Soluble adhesives, ${ }^{3}$ i.e. the powder and paste forms, are universally accepted now because they do not damage the soft tissues. The use of dental adhesives began in the XVIII 
Table 1: Comparison of two groups, i.e. resorbed ridge and good (high well-rounded) ridge with respect to retention without adhesive, retention with adhesive (fittydent) and retention with adhesive (fixon) by t-test

\begin{tabular}{llllll}
\hline Retention & Groups & Mean & $S D$ & $t$-value & $p$-value \\
\hline Without adhesive & Resorbed ridge & 9.93 & 2.27 & -16.8328 & $0.00001^{*}$ \\
& Good (high well-rounded) ridge & 24.53 & 4.17 & & \\
With adhesive (fittydent) & Resorbed ridge & 29.56 & 2.93 & -3.8958 & $0.0003^{*}$ \\
& Good (high well-rounded) ridge & 33.05 & 3.92 & & \multirow{2}{*}{$0.00001^{*}$} \\
With adhesive (fixon) & Resorbed ridge & 16.47 & 3.02 & -11.8448 & \\
& Good (high well-rounded) ridge & 27.70 & 4.23 & & \\
\hline
\end{tabular}

${ }^{*} p<0.05$

Table 2: Comparison of retention without adhesive, retention with adhesive (fittydent) and retention with adhesive (fixon) in resorbed (low well-rounded) ridge group by paired t-test

\begin{tabular}{lllllll}
\hline Retention (in Newton's) & Mean & Std. dev. & Mean diff. & SD diff. & Paired t-test & $p$-value \\
\hline Without adhesive & 9.93 & 2.27 & -19.63 & 1.93 & -55.6708 & $0.00001^{*}$ \\
With adhesive (fittydent) & 29.56 & 2.93 & & & & \\
Without adhesive & 9.93 & 2.27 & -6.54 & 1.57 & -22.7927 & $0.00001^{*}$ \\
With adhesive (fixon) & 16.47 & 3.02 & & & \multirow{2}{*}{36.3380} & $0.00001^{*}$ \\
With adhesive (fittydent) & 29.56 & 2.93 & 13.09 & 1.97 & & \\
With adhesive (fixon) & 16.47 & 3.02 & & &
\end{tabular}

${ }^{*} p<0.05$

Table 3: Comparison of retention without adhesive, retention with adhesive (fittydent) and retention with adhesive (fixon) in good (high well-rounded) ridge group by paired t-test

\begin{tabular}{lllllll}
\hline Retention (in Newton's) & Mean & Std. dev. & Mean diff. & SD diff. & Paired t-test & $p$-value \\
\hline Without adhesive & 24.53 & 4.17 & -8.52 & 1.03 & -45.3342 & $0.00001^{*}$ \\
With adhesive (fittydent) & 33.05 & 3.92 & & & & \\
Without adhesive & 24.53 & 4.17 & -3.17 & 0.94 & -18.5881 & $0.00001^{*}$ \\
With adhesive (fixon) & 27.70 & 4.23 & & & \\
With adhesive (fittydent) & 33.05 & 3.92 & 5.34 & 1.32 & 22.2311 & $0.00001^{*}$ \\
With adhesive (fixon) & 27.70 & 4.23 & & & \\
\hline
\end{tabular}

${ }^{*} p<0.05$

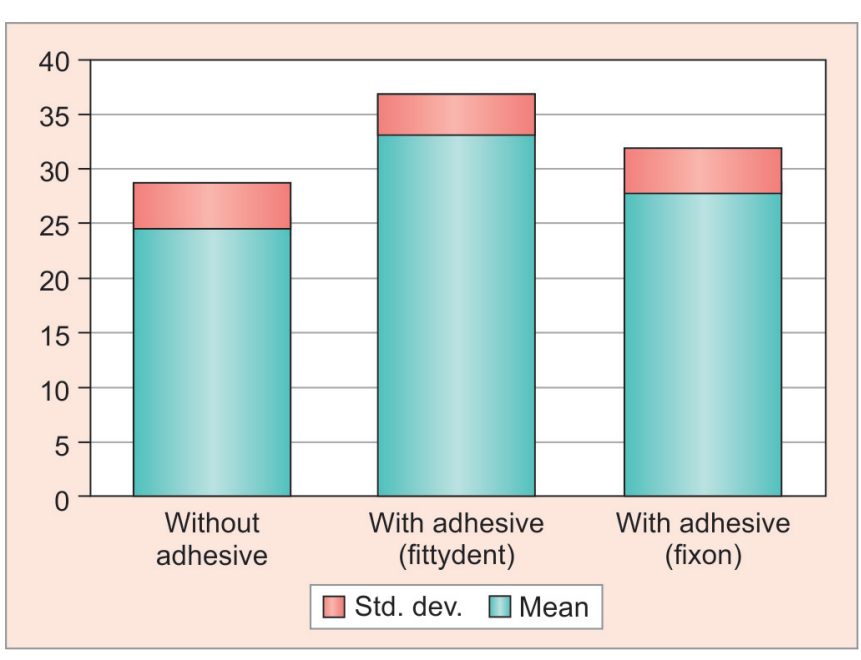

Graph 3: Comparison of retention without adhesives, retention with adhesive (fittydent) and retention with adhesive (fixon) in high well-rounded ridges

century. ${ }^{4}$ These products were prepared by pharmacists who mixed plant gums to produce a material that could absorb the humidity of saliva and swell to form a mucilaginous layer adhering to the oral mucosa and dentures. Shay ${ }^{5}$ described the mechanism of action of adhesives in 1991. These materials swell 50 to $150 \%$ by volume in the presence of water, filling the spaces between the prosthesis and the tissues. Saliva increases the viscosity of the adhesive thereby increasing the force required to separate the prosthesis from the tissue surface.

Adhesives have gained importance and popularity because of the increase in their demand owing to the increased geriatric population. Complete dentures still represent one of the most important treatment options in prosthodontics, especially in the Indian scenario. Adequate retention and stability are the prerequisites in the success of the complete denture therapy. Therefore, improving retention and stability is of considerable interest in prosthodontics. Traditional literature stresses on the ill effects of denture adhesives since the side effects have been well-documented, i.e.:

- I: Enhancing the prolonged use of ill fitting dentures thus promoting residual ridge resorption.

- II: Interfering with the occlusion as a result of uneven and uncontrolled thickness of intervening adhesive.

- III: Act as allergens. ${ }^{6}$

Therefore, in an attempt to solve this dilemma regarding the use of denture adhesives, a survey was conducted among the academic prosthodontists and it was concluded that denture adhesives are an useful adjunct in denture prosthesis 
service. Education for both dentists and the patients is imperative for the proper use and avoid misuse of the same. (Slaughter Ann et al 1999). ${ }^{7}$

The current dental literature supports the use of denture adhesives. In the elderly, the psychological management of the patient is as important as the technical management so denture adhesives may actually be a boon as far as the psychological satisfaction of the patient is concerned. Hasegawa et $\mathrm{al}^{8}$ estimated the retention and stability of dentures after using adhesives in three dimensional as well as rotational movements and subsequently its effect on chewing function. The results indicated that denture adhesives contributed to reducing denture movement thus improving function.

The aim of the present study was to assess the efficacy of some very commonly available adhesives namely fixon and fittydent powders on different conditions of denture bearing tissues. Powder forms were particularly tested as they were seen to be more readily available over the counter materials and also they were found to be used more commonly used by denture wearers. The results of the present study showed that the use of the denture adhesives not only improved retention in patients with good ridges but patients with poor foundations were also benefitted (Table 1 and Graph 1). This was in accordance with the study conducted by Fujimori et $\mathrm{al}^{9}$ on the effects of denture adhesives on the masticatory functions of complete denture wearers considering different conditions of denture bearing tissues. The use of denture adhesives improved maximum biting force and increased provided rhythmic masseter muscle activity during mastication for all patients, but masticatory function improvement was more significant for denture wearers with poor denture bearing tissues than good denture bearing tissues.

Abdulla and Khamas, ${ }^{10}$ in their study, revealed that there is a significant improvement in the retention of mandibular complete denture for flat ridge after using three types' denture adhesives. Denture adhesives start their action immediately and decreased with time. Powder was the most effective denture adhesives than cushion and cream.

In the present study, the retentive ability of two commonly available denture adhesive powders was evaluated in vivo.

The retentive ability of denture adhesives presented a much greater retentive force than that of saliva alone, i.e. when no adhesive was used. Among the various denture adhesives tested, fittydent exhibited better results (Tables 2 and 3, Graphs 2 and 3) although both adhesives showed significant resistance to dislodgement when the test apparatus was applied (Table 1 and Graph 1).

\section{CONCLUSION}

The following conclusions can be drawn from the study:

1. All denture adhesives investigated in this study exhibited significantly higher retentive ability than saliva.

2. Significant improvement in retention was observed in patients with poor foundations as well as patient with good supporting tissues.

3. Among the denture adhesive powders tested, fittydent powder offered greater resistance to dislodgement.

The use of adhesives has always been under scrutiny and has been surveyed extensively. In general, most of the patients responded positively indicating that the retention was little better or much better with the use of adhesive materials. ${ }^{11}$

In consequence, to the results of this study it can be said that denture adhesives do increase the retentive ability of a removable prosthesis and should be suggested to the difficult patients like patients with systemic diseases like Parkinson's disease, Alzheimers disease, in maxillofacial surgery patients for obturators, in public speakers like attorneys, vocalists, etc. Nevertheless, keeping in mind the problems encountered due to the usage of these materials dentists should caution their patients to limit the use of denture adhesives in accordance with manufacturers' instructions. These materials are readily available over the counter products, this should be discouraged and such materials should be made available only on doctors prescriptions.

\section{REFERENCES}

1. Atwood DA. Reduction of residual ridges: a major oral disease entity. J Prosthet Dent 1971;26:280-295.

2. Zarb GA, Bolender Cl. Boucher's prosthodontic treatment of the edentulous patient. 11th ed. St Louis: Mosby 1997;442.

3. Adisman IK. The use of denture adhesives as an aid to denture treatment. J Prosthet Dent 1989;62:711-715.

4. McCabe JF, Carrick TE, Kamohara H. Adhesive bond strength and compliance for denture soft lining materials. Biomaterials 2002;23:1347-1352.

5. Shay K. Denture Adhesives - choosing the right powders and pastes. J Am Dent Assoc 1991;70-76.

6. Panagiotouni E, Pissiotis A, Kapari D, Kaloyannides NN. Retentive ability of various denture adhesive materials: an in vitro study. J Prosthet Dent 1995;73:578-585.

7. Slaughter A, Katz RV, Grasso JE. Professional attitudes toward denture adhesives: a Delphi technique survey of academic prosthodontics. J Prosthet Dent 1999;82:80-89.

8. Hasegawa S, Sekita T, Hayakawa I. Effect of denture adhesive on stability of complete dentures and the masticatory function. J Med Dent Sci 2003;50:239-247.

9. Fujimori T, Hirano S, Hayakawa I. Effects of a denture adhesive on masticatory functions for complete denture wearers. Consideration for the condition of denture-bearing tissues. J Med Dent Sci 2002;49:151-156. 
10. Ilham H,Al-Abdullah, Khamas AM. The effect of three different denture adhesives on the retention of mandibular complete denture (comparative study). J Bagh Coll Dent 2009;21(2): 5-9.

11. Kesley CC, Lang BR, Wang RF. Examining patients response about the effectiveness of five denture adhesive pastes. J Am Dent Assoc 1997;128:1532-1538.

\section{ABOUT THE AUTHORS}

\section{Puja Malhotra (Corresponding Author)}

Reader, Department of Prosthodontics, SGT Dental College, Gurgaon

Haryana, India, e-mail: pujchow@yahoo.com

\section{Bhupender Yadav}

Senior Lecturer, Department of Prosthodontics, SGT Dental College Gurgaon, Haryana, India

\section{Harish Yadav}

Professor, Department of Prosthodontics, SGT Dental College, Gurgaon Haryana, India

\section{Sumit Singh Phukela}

Reader, Department of Prosthodontics, SGT Dental College, Gurgaon Haryana, India 